\title{
Coupling enhancement of split ring resonators on graphene
}

\author{
Semih Cakmakyapan ${ }^{a, c, *}$, Humeyra Caglayan ${ }^{b, c}$, Ekmel Ozbay ${ }^{a, c, d}$ \\ a Department of Physics, Bilkent University, 06800 Ankara, Turkey \\ ${ }^{\mathrm{b}}$ Electrical and Electronics Department, Abdullah Gul University, Kayseri, Turkey \\ c Nanotechnology Research Center, Bilkent University, 06800 Ankara, Turkey \\ d Department of Electrical and Electronics Engineering, Bilkent University, 06800 Ankara, Turkey
}

\section{A R T I C L E I N F O}

Article history:

Received 17 April 2014

Accepted 19 August 2014

Available online 27 August 2014

\begin{abstract}
A B S T R A C T
Metallic split ring resonator (SRR) structures are used in nanophotonics applications in order to localize and enhance incident electromagnetic field. Electrically controllable sheet carrier concentration of graphene provides a platform where the resonance of the SRRs fabricated on graphene can be tuned. The reflectivity spectra of SRR arrays shift by applying gate voltage, which modulates the sheet carrier concentration, and thereby the optical conductivity of monolayer graphene. We experimentally and numerically demonstrated that the tuning range can be increased by tailoring the effective mode area of the SRR and enhancing the interaction with graphene. The tuning capability is one of the important features of graphene based tunable sensors, optical switches, and modulator applications.
\end{abstract}

(c) 2014 Elsevier Ltd. All rights reserved.

\section{Introduction}

Nanophotonics is a growing research field, which leads to potential optical devices that can control and manipulate light on the nanometer scale [1]. The strong interaction of light with metallic nanostructures and their optical response have been studied and have led to numerous applications [2]. An important class of metallic nanostructures is the split ring resonator (SRR), which was originally proposed by Pendry et al. in order to create the desired susceptibility [3]. SRRs can produce an effect of being electrically smaller when responding to an oscillating electromagnetic field and support resonance wavelengths much smaller than their physical sizes. Therefore, they are able to concentrate the electric field in a small volume and, furthermore, enhance the electric field [4,5].

Recently, graphene has attracted a great deal of attention due to its two-dimensional monolayer structure and uncon- ventional electrical [6], and plasmonic properties [7-9]. Graphene has been a very promising material for photonic devices such as photodetectors [10,11], polarizers [12], and tunable optical antennas $[13,14]$. Papasimakis et al. experimentally showed the optical response due to graphene on metamaterial [15] and later Zou et al. demonstrated the electromagnetic interaction between graphene and metamaterials theoretically [16]. One of the most interesting optical properties of graphene for nanophotonic applications is the tunability of optical conductivity, which depends on both interband and intraband transitions [17]. Intraband transitions are sensitive to the change in Fermi energy, $E_{F}$, which is controlled via electrostatic doping, and intraband transitions dominate, when $2 E_{F}>\hbar \omega$, where $\hbar \omega$ is the photon energy. Since the optical conductivity of graphene can be changed by electrostatic doping, graphene is a strong candidate for tunable plasmonic devices $[13,18]$. However, it is a challenge to use graphene in

\footnotetext{
* Corresponding author at: Nanotechnology Research Center, Bilkent University, 06800 Ankara, Turkey.

E-mail address: semihc@bilkent.edu.tr (S. Cakmakyapan). 
optoelectronic devices due to its weak interaction with light. In spite of its electrically tunable nature, the modulation of the optical response can be limited $[19,20]$. This problem can be overcome by introducing metallic nanostructures, which enhance light-graphene interactions within larger interaction volumes [21,22].

In this paper, we have experimentally and numerically shown that the tunability range of SRRs on graphene is enhanced by increasing the interaction volume between graphene and the localized electric field inside the gap region. By designing SRRs that operate at mid-infrared wavelengths with different effective mode areas, we have shown that the reflection spectra of these resonators can be shifted with applied voltage. Moreover, the light-graphene interaction determines the tunability range, and it can be tailored by increasing the effective mode area.

\section{Methods}

In this study, CVD grown monolayer graphene on $\mathrm{SiO}_{2}$ deposited silicon substrate is used, which is provided from the Graphene Supermarket. A schematic of the device is shown in Fig. 1(a). SRRs are fabricated on the graphene (shown in purple) sample by electron beam lithography. The metal thickness of the gold SRRs is $50 \mathrm{~nm}$. The devices on the sample are isolated from each other by etching the graphene with oxygen plasma. Later, large contact pad pairs are fabricated by photolithography, and $20 / 200 \mathrm{~nm}$ Ti/Au metals, which function as source and drain contacts, are evaporated for probing and bonding during the measurements. Finally, a Ti/Au metal pair is evaporated on the backside of the sample in order to be used as a back-gate. Gate voltage is applied between graphene and p-type silicon substrate, which are separated by $285 \mathrm{~nm}$ thick $\mathrm{SiO}_{2}$ (shown in blue). Two different types of resonators, SRR-1 and SRR-2 are studied. Their dimensions and SEM images are shown in Fig. 1(b) and (c). The only difference between the two structures is that SRR2 has a larger gap area. The dimensions of the SRRs are $w=70 \mathrm{~nm}, u=400 \mathrm{~nm}, g=40 \mathrm{~nm}$, and $h=120 \mathrm{~nm}$; and the period of the arrays is $600 \mathrm{~nm}$ in both directions.
The current-voltage (I-V) characteristics of graphene devices are given in Fig. 2. Fig. 2(a) shows that the current flowing on graphene is modulated by varying the gate voltage. The charge neutrality point of graphene is measured as $\mathrm{V}_{\mathrm{CNP}}=130 \mathrm{~V}$. I-V measurements between the source and drain contacts are performed. The results in Fig. 2(b) show good ohmic characteristics with a constant resistance around $1.2 \mathrm{k} \Omega$, which is a typical value for graphene samples [23]. Additionally, the mobility and the sheet carrier concentration of graphene are $\mu=2153 \mathrm{~cm}^{2} / \mathrm{Vs}$ and $\mathrm{N}=5.6 \times 10^{12} \mathrm{~cm}^{-2}$, respectively, which are obtained by performing Hall measurements. Capacitance per unit area of the devices can be calculated by $C=\mathrm{Ne} / \mathrm{V}$ [24], which results as $C=69 \mathrm{aF} / \mu \mathrm{m}^{2}$.

Finite-difference time domain (FDTD) simulations are carried out using the commercially available software package Lumerical FDTD Solutions. Graphene is introduced as a $1 \mathrm{~nm}$ thick dispersive material by using the optical conductivity formula given in Eq. (1) $[25,26]$, where $k_{B}$ is the Boltzmann constant, $T$ is the temperature, $\omega$ is the frequency, $E_{F}$ is the Fermi energy, and $\tau$ is the carrier relaxation lifetime.

$$
\begin{aligned}
\sigma_{s}(\omega)= & \frac{2 i e^{2} k_{B} T}{\pi \hbar^{2} \omega+i / \tau} \ln \left[2 \cosh \left(\frac{E_{F}}{2 k_{B} T}\right)\right] \\
& +\frac{e^{2}}{4 \hbar}\left[\frac{1}{2}+\frac{1}{\pi} \arctan \left(\frac{\hbar \omega-2 E_{F}}{2 k_{B} T}\right)\right. \\
& \left.-\frac{i}{2 \pi} \ln \left(\frac{\left(\hbar \omega+2 E_{F}\right)^{2}}{\left(\hbar \omega-2 E_{F}\right)^{2}+4\left(k_{B} T\right)^{2}}\right)\right]
\end{aligned}
$$

The carrier relaxation lifetime, $\tau$, is calculated by using Eq. (2). Here $\sigma=n e \mu$ is semi-classical diffusive conductivity for 2D graphene, and $g_{s}=g_{v}=2$ are the spin and valley degeneracy factors [27].

$$
\tau=\sigma \frac{h}{g_{s} g_{v} e^{2}} \frac{2 \hbar}{E_{F}}
$$

The optical conductivity of graphene depends on Fermi energy, $E_{F}=\hbar v_{F} \sqrt{\pi n}$, which is a function of the sheet carrier concentration. Fermi energy, and thereby the sheet carrier concentration, can be changed by applying gate voltage, which is linearly proportional to the sheet carrier concentration $[24,28]$. As a result, the optical conductivity of graphene

(a)
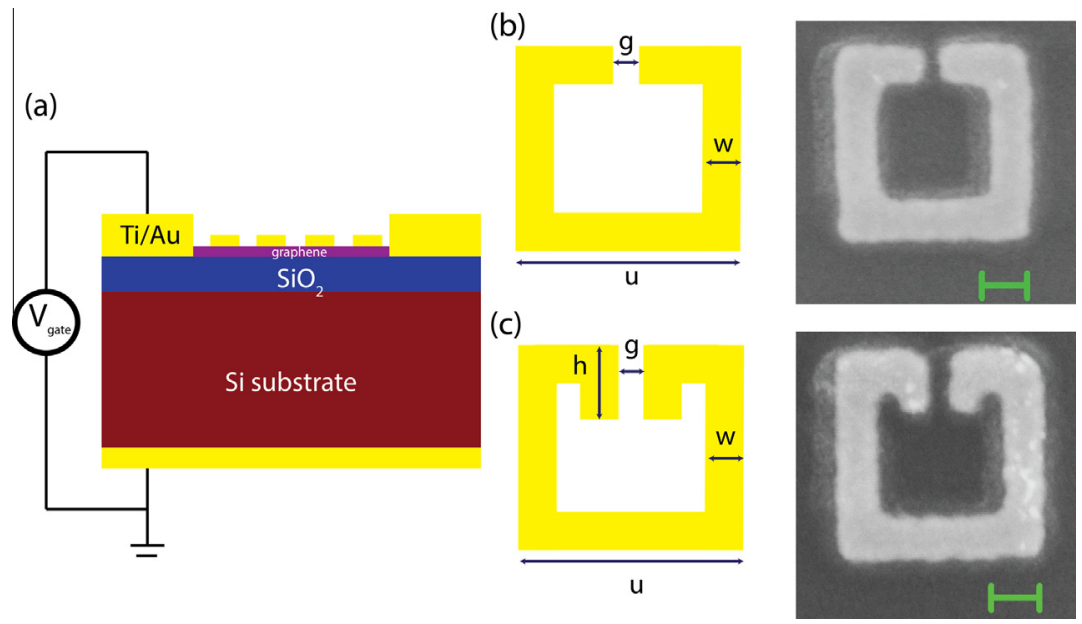

Fig. 1 - (a) Cross-section view of the tunable SRR device; schematics and SEM image of (b) SRR-1, and (c) SRR-2 structures, scale bar is $100 \mathrm{~nm}$. $w=70 \mathrm{~nm}, u=400 \mathrm{~nm}, g=40 \mathrm{~nm}$, and $h=120 \mathrm{~nm}$. (A color version of this figure can be viewed online.) 

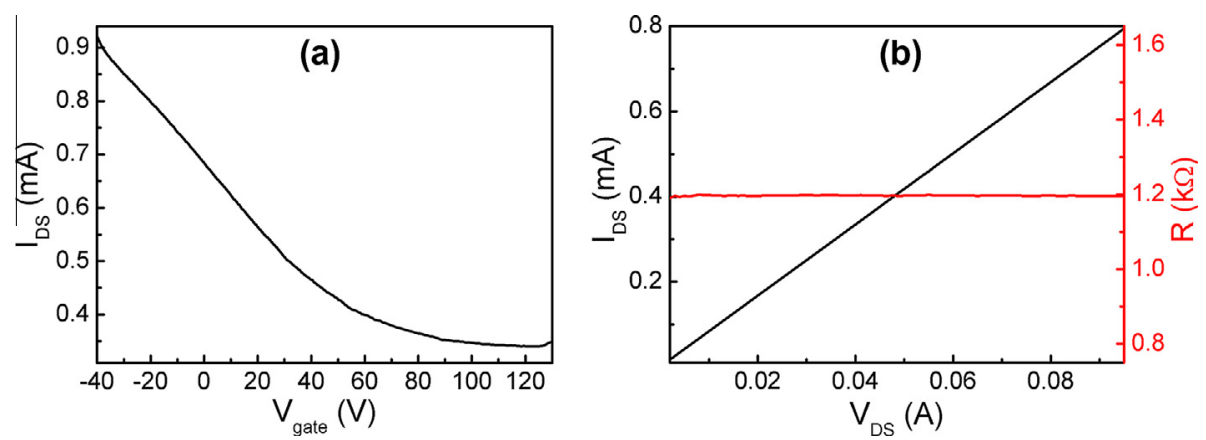

Fig. 2 - DC-IV measurements: (a) current between drain and source under applied gate voltage, (b) current vs. voltage dependence and resistance of graphene. (A color version of this figure can be viewed online.)

can be tuned at different gate voltages. Permittivity of graphene can be obtained for all Fermi energy values by implementing Eq. (3).

$\varepsilon(\omega)=1+\frac{i \sigma_{s}}{\omega \varepsilon_{0} t_{G}}=\varepsilon_{r}+i \varepsilon_{i}$

\section{Results and discussion}

The measured and calculated reflectivity results for SRR-1 are demonstrated in Fig. 3(a) and (b). The reflectivity measurements are taken by using Fourier transform infrared (FTIR) spectroscopy at different gate voltages in order to investigate the resonance behavior for different sheet carrier concentrations. Here the voltage difference, $\Delta \mathrm{V}$, is defined by $\Delta \mathrm{V}=$ $\mathrm{V}_{g}-\mathrm{V}_{\mathrm{CNP}}$, where $\mathrm{V}_{g}$ is the gate voltage, and $\mathrm{V}_{\mathrm{CNP}}=130 \mathrm{~V}$ is the voltage at the charge neutrality point, as shown in Fig. 2(a). $\Delta \mathrm{V}=-170 \mathrm{~V}$ corresponds to the highest doped case, and hence the highest Fermi energy for graphene. Since the refractive index of the graphene layer, $n=\sqrt{\varepsilon(\omega)}$, changes for each different gate voltage according to Eq. (3), the reflectivity spectrum shifts when the gate voltage is varied. It is seen in both experiment and simulation results that the resonance peak shifts to longer wavelengths as the doping and thus the Fermi energy of the graphene decreases. $62 \mathrm{~nm}$ red shift is measured, as the gate voltage approaches the charge neutrality point, $\Delta \mathrm{V}=0 \mathrm{~V}$. The corresponding simulation results show that this shift is $130 \mathrm{~nm}$, as shown in Fig. 3(b).

Fig. 4 shows the reflectivity spectra for SRR-2 array. In this case, $95 \mathrm{~nm}$ red-shift in experiments, and $160 \mathrm{~nm}$ shift in simulations are obtained. In addition to resonance peaks, curves shift asymmetrically, where the reflection curve at the charge neutrality point becomes the broadest one with the lowest quality factor. The reason is that the optical conductivity of graphene is more sensitive at longer wavelengths, where the intraband transitions dominate. Simulation results show larger wavelength shifts than the experiment results for both structures, as demonstrated in Figs. 3 and 4(b). The possible reasons of this discrepancy can be due to the fabrication imperfections, the defects, and grain boundaries on graphene.

Moreover, resonance peaks at each gate voltage is investigated, and the results for SRR-1 and SRR-2 structures are demonstrated in Fig. 5(a) and (b), respectively, where the peak-to-peak difference keeps increasing as the doping of the graphene is increased. The results show that SRR-2 exhibits a larger tunability range in reflection spectra compared to SRR-1. Electric field distributions at the resonance wavelengths for SRR-1 and SRR-2 are demonstrated in Fig. 5(c) and (d). It is clearly seen that the field is highly localized at the gap region for the polarization indicated in the figures, since the gap of these SRR structures can be excited with $x$ polarized light.

The gap region plays an important role, since the field is localized inside the gap at the resonance wavelength. Increasing the light-graphene interaction area, as in the case for the SRR-2 structure, results in larger shifts, since the effective mode area is larger compared to SRR-1. For further investigation, effective mode area inside the gap region is calculated for both of the structures. The effective mode area is the ratio between the total energy density per unit length and the peak energy density of the mode. According to this calculation, the

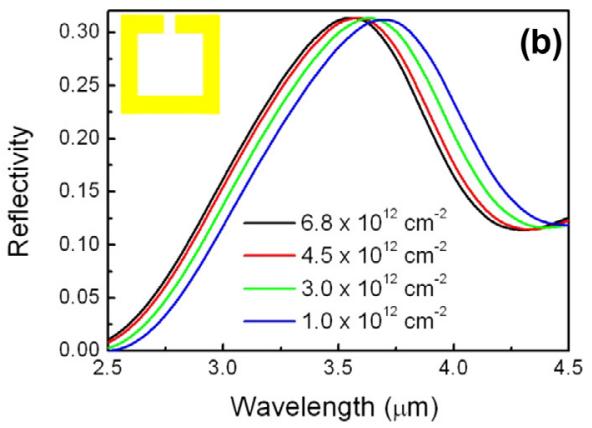

Fig. 3 - Reflectivity spectra of SRR-1 structure (a) experiment, (b) simulation results. The resonance peak shifts to longer wavelengths. (A color version of this figure can be viewed online.) 

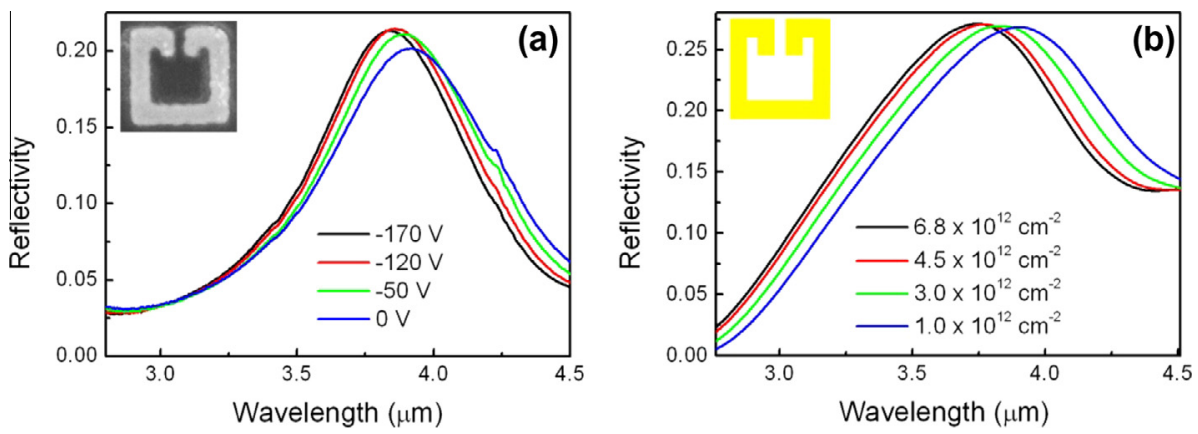

Fig. 4 - Reflectivity spectra of SRR-2 structure (a) experiment, (b) simulation results. The resonance wavelength shifted $95 \mathrm{~nm}$. (A color version of this figure can be viewed online.)

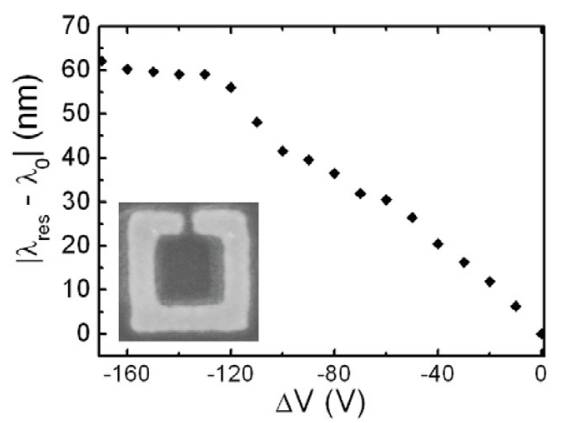

(a)

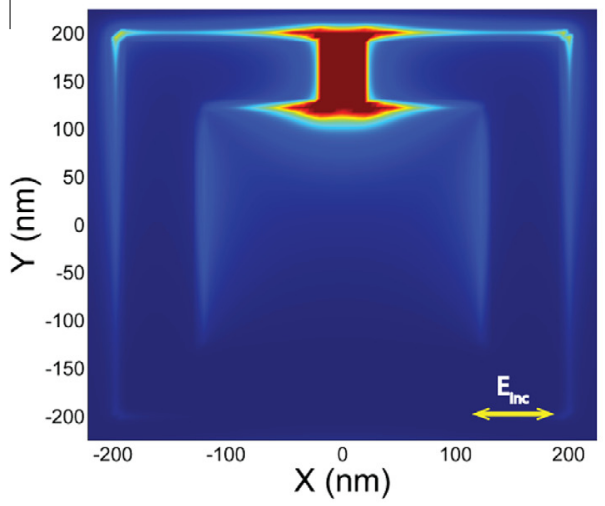

(c)

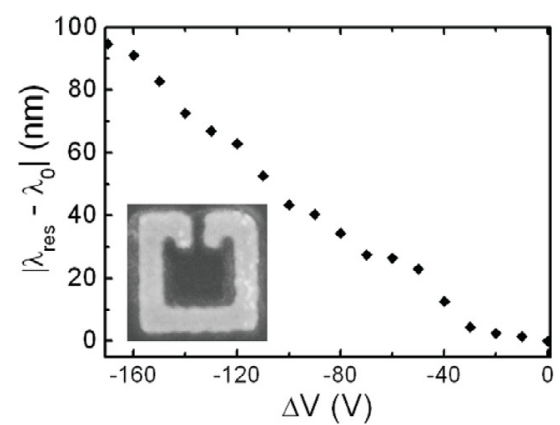

(b)

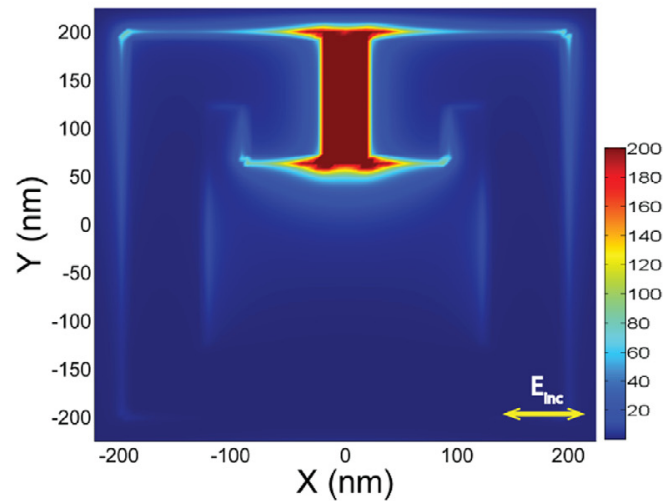

(d)

Fig. 5 - Resonance wavelength shift with respect to the reflectivity measurement taken at the charge neutrality point for (a) SRR-1, and (b) SRR-2; $\lambda_{\text {res }}$ represents the resonance wavelength, and $\lambda_{0}$ is the resonance wavelength at the charge neutrality point, $\Delta \mathrm{V}=0 \mathrm{~V}$, so that the difference $\left|\lambda_{\text {res }}-\lambda_{0}\right|$ gives the shift with respect to the undoped graphene. Electric field distributions at resonance frequencies for (c) SRR-1 at $3.5 \mu \mathrm{m}$, (d) SRR-2 at $3.9 \mu \mathrm{m}$; the maximum of the color bar is set to the same value in both figures for comparison, and the electric field is in the $x$-direction. (A color version of this figure can be viewed online.)

effective mode area of SRR-1 is $1100 \mathrm{~nm}^{2}$, whereas the effective mode area for SRR-2 is $2010 \mathrm{~nm}^{2}$.

\section{Conclusion}

In conclusion, we demonstrated that the optical response of split ring resonators on graphene can be tuned with a transistor-like device, where the optical conductivity of graphene is modulated by varying the gate voltage. Furthermore, the tuning range can be increased by designing the structures such that the effective mode area between graphene and the localized field becomes larger. The experiment and the simulation results showed a good agreement. It can be foreseen that the shift at the resonance can be increased with a structure having an effective mode area larger than our proposed SRRs. In addition, the structures can be optimized to operate at even longer wavelengths by scaling up. It is possible to use such devices as tunable sensors, optical switches and modulators.

\section{Acknowledgements}

The authors would like to thank to Francesco Pierini for the discussions held on structure designs. This work is supported 
by the projects DPT-HAMIT, DPT-FOTON, NATO-SET-193 and TUBITAK under Project Nos., 113E331, 109A015, $109 E 301$. One of the authors (E.O.) also acknowledges partial support from the Turkish Academy of Sciences.

\section{R E F E R E N C E S}

[1] Atwater HA. The promise of plasmonics. Sci Am 2007;296:56-63.

[2] Lindquist NC, Nagpal P, McPeak KM, Norris DJ, Oh SH. Engineering metallic nanostructures for plasmonics and nanophotonics. Rep Prog Phys 2012;75.

[3] Pendry JB, Holden AJ, Robbins DJ, Stewart WJ. Magnetism from conductors and enhanced nonlinear phenomena. IEEE Trans Microw Theory 1999;47:2075-84.

[4] Shelby RA, Smith DR, Schultz S. Experimental verification of a negative index of refraction. Science 2001;292:77-9.

[5] Aydin K, Bulu I, Guven K, Kafesaki M, Soukoulis CM, Ozbay E. Investigation of magnetic resonances for different split-ring resonator parameters and designs. New J Phys 2005;7.

[6] Geim AK, Novoselov KS. The rise of graphene. Nat Mater 2007;6:183-91.

[7] de Abajo FJG. Graphene plasmonics: challenges and opportunities. ACS Photon 2014;1:135-52.

[8] Koppens FHL, Chang DE, de Abajo FJG. Graphene plasmonics: a platform for strong light-matter interactions. Nano Lett 2011;11:3370-7.

[9] Bao QL, Loh KP. Graphene photonics, plasmonics, and broadband optoelectronic devices. ACS Nano 2012;6:3677-94.

[10] Zhang YZ, Liu T, Meng B, Li XH, Liang GZ, Hu XN, et al. Broadband high photoresponse from pure monolayer graphene photodetector. Nat Commun 2013;4.

[11] Xia FN, Mueller T, Lin YM, Valdes-Garcia A, Avouris P. Ultrafast graphene photodetector. Nat Nanotechnol 2009;4:839-43.

[12] Bao QL, Zhang H, Wang B, Ni ZH, Lim CHYX, Wang Y, et al. Broadband graphene polarizer. Nat Photon 2011;5:411-5.

[13] Yao Y, Kats MA, Genevet P, Yu NF, Song Y, Kong J, et al. Broad electrical tuning of graphene-loaded plasmonic antennas. Nano Lett 2013;13:1257-64.
[14] Fang ZY, Wang YM, Schather AE, Liu Z, Ajayan PM, de Abajo FJG, et al. Active tunable absorption enhancement with graphene nanodisk arrays. Nano Lett 2014;14:299-304.

[15] Papasimakis N, Luo ZQ, Shen ZX, De Angelis F, Di Fabrizio E, Nikolaenko AE, et al. Graphene in a photonic metamaterial. Opt Expr 2010;18:8353-9.

[16] Zou YH, Tassin P, Koschny T, Soukoulis CM. Interaction between graphene and metamaterials: split rings vs. wire pairs. Opt Expr 2012;20:12198-204.

[17] Kim J, Son H, Cho DJ, Geng BS, Regan W, Shi SF, et al. Electrical control of optical plasmon resonance with graphene. Nano Lett 2012;12:5598-602.

[18] Fang ZY, Liu Z, Wang YM, Ajayan PM, Nordlander P, Halas NJ. Graphene-antenna sandwich photodetector. Nano Lett 2012;12:3808-13.

[19] Emani NK, Chung TF, Ni XJ, Kildishev AV, Chen YP, Boltasseva A. Electrically tunable damping of plasmonic resonances with graphene. Nano Lett 2012;12:5202-6.

[20] Cakmakyapan S, Sahin L, Pierini F, Strupinski W, Ozbay E. Resonance broadening and tuning of split ring resonators by top-gated epitaxial graphene on SiC substrate. Appl Phys Lett 2013;103.

[21] Yao Y, Kats MA, Shankar R, Song Y, Kong J, Loncar M, et al. Wide wavelength tuning of optical antennas on graphene with nanosecond response time. Nano Lett 2014;14:214-9.

[22] Li ZY, Yu NF. Modulation of mid-infrared light using graphene-metal plasmonic antennas. Appl Phys Lett 2013;102.

[23] Russo S, Craciun MF, Yamamoto M, Morpurgo AF, Tarucha S. Contact resistance in graphene-based devices. Phys E 2010;42:677-9.

[24] Li ZQ, Henriksen EA, Jiang Z, Hao Z, Martin MC, Kim P, et al. Dirac charge dynamics in graphene by infrared spectroscopy. Nat Phys 2008;4:532-5.

[25] Falkovsky LA, Pershoguba SS. Optical far-infrared properties of a graphene monolayer and multilayer. Phys Rev B 2007;76.

[26] Falkovsky LA, Varlamov AA. Space-time dispersion of graphene conductivity. Eur Phys J B 2007;56:281-4.

[27] Adam S, Hwang EH, Galitski VM, Das Sarma S. A selfconsistent theory for graphene transport. Proc Natl Acad Sci U S A 2007;104:18392-7.

[28] Novoselov KS, Geim AK, Morozov SV, Jiang D, Katsnelson MI, Grigorieva IV, et al. Two-dimensional gas of massless Dirac fermions in graphene. Nature 2005;438:197-200. 